\title{
Medical error reporting: is it about physicians' knowledge and their practice, or patient safety culture in the workplace?
}

In their original article published in the Eastern Mediterranean Health Journal entitled "Physicians' knowledge and practice towards medical error reporting: a cross-sectional hospital-based study in Saudi Arabia" Alsafi et al. used a questionnaire to assess physicians' knowledge and their practice in reporting medical errors (1). However, it is not clear how physicians' knowledge related to medical error reporting, nor it is clear how the reported physician's practices could provide the actual reasons for under-reporting or minimizing the recurrence of avoidable patterns of higher error rates. The design of the work raises a number of issues.

First, one would wonder about the validity of the questionnaire used and how the questionnaire was constructed. There are no references to show if the questionnaire was adopted from the literature, nor there were justifications for the questions included in the questionnaire. Questions about "most frequently" and "most common" medical errors in the hospital represented $50 \%$ of questions about physicians' knowledge.
These questions are difficult to answer, and encourage guessing, particularly for staff who have recently joined the medical workforce or are recently appointed to the hospital.

Second, there were no questions about whether the staff were trained in the area of patient safety and reporting medical errors, nor there were questions on the use of technology, information accessibility, communication skills, patient collaboration, multi-professional teamwork or type of culture embedded in the hospital.

Third, in the discussion the authors indicated that, "in Canada, error discloser is not explicitly addressed in the new Canadian Medical Association's Code of Ethics. In addition, most professional bodies, such as the College of Physicians and Surgeons of Ontario, the Province's regulatory body for physicians, have no policies requiring physicians to disclose errors except in some circumstances...". The reference cited by the authors dated back to 2001. Such statements do not reflect current Canadian Medical Association (CMA) patient safety policy framework updated in 2010 (2). CMA in its policy stated, "patient safety initiatives should encourage and anticipate the full and appropriate disclosure to patients of relevant information that is material to their health and healthcare, including information about adverse events or effects". Also the College of Physicians and Surgeons of Ontario in its recent document (3), clearly stated the regulations and procedures for adverse events reporting. Furthermore, the Canadian Patient Safety Institute has on its website documents on patient safety and medical error reporting recommendation procedures (4) that the authors did not refer to.

One would wonder if physicians' knowledge was the right target and how such information could improve performance. New approaches in this area aim at building a systems approach, the development of a culture of patient safety and introducing strategies to minimize the recurrence of avoidable patterns of medical errors and poor reporting (5).

\section{References}

1. Alsafi E, Baharoon S, Ahmed A, Al-Jahdali HH, Al Zahrani S, Al Sayyari A. Physicians' knowledge and practice towards medical error reporting: a cross-sectional hospital-based study in Saudi Arabia. East Mediterr Health J. 2015 Oct 2;21(9):655-64. PMID:26450862

2. CMA patient safety policy framework (updated 2010). Ottawa (ON): Canadian Medical Association; 2010 (https://www.cma. $\mathrm{ca} /$ Assets/assets-library/document/en/advocacy/policyresearch/CMA_Policy_CMA_Patient_Safety_Policy_Framework_Update_2010_PD10-04-e.pdf\#search=patient\%20 safety, accessed 5 November 2015).

3. Matlow A, Brian S. A culture of patient safety: foundation for a Royal College patient safety roadmap. Ottawa (ON): Royal
College of Physicians and Surgeons of Canada; 2014 (http:// www.royalcollege.ca/portal/page/portal/rc/common/documents/policy/culture_of_patient_safety.pdf; accessed 5 November 2015).

4. Canadian Patient Safety Institute [Internet] (http://www. patientsafetyinstitute.ca/en/education/Pages/default.aspx, accessed 5 November 2015).

5. La Pietra L, Calligaris L, Molendini L, Quattrin R, Brusaferro S. Medical errors and clinical risk management: state of the art. Acta Otorhinolaryngol Ital. 2005 Dec;25(6):339-46. PMID:16749601

S.A. Azer

Professor, Medical Education Department, King Saud University, Riyadh, Saudi Arabia (Azer2000@optusnet.com.au) 


\section{Author response}

To start I would like to thank you for your interest in reading our paper "Physicians' knowledge and practice towards medical error reporting: a cross-sectional hospital-based study in Saudi Arabia" and raising these legitimate questions.

Physicians' knowledge of the occurrence of medical errors is an important recognition step towards better reporting practices. If physicians underestimated the occurrence of errors, a false safety environment may be created.

It is very important to distinguish between the maturity of different health care systems when it comes to reporting errors. While the concepts of patient safety emerged in the United States and Europe many years ago, developing countries have only started to build this culture. Transparency in medical error disclosure is a key element in patient safety. Physician's actual practices of medical error reporting are influenced by their knowledge of the existing system of disclosing medical errors.

We acknowledge that questions and concerns raised are important to be asked and addressed. Many of the patient safety concepts are relatively newly embedded in the health care heritage in Saudi Arabia. We do not expect that currently the majority of our workforce is exposed to them, even less so at the time when our work was done.

We acknowledge some of the limitations of the questionnaire used, however we feel the questions were appropriate for the research aims. As evident by our results, we did not have many respondents who had only just joined the hospital. We support more streamlining of the questionnaire in future study to include all further elements of missing information.

We also acknowledge the observation that the reference related to the Canadian Medical Association's Code of Ethics should have included the updated patient safety policy framework of 2010. The update includes encouragement for physicians for what they refer as "appropriate disclosure "of adverse events or effects. We feel that more emphasis and mandating regulations can be further built in the current document.

We are very grateful for the enlightening remarks raised and we will be sure to bear them in mind them for our future research. 